\title{
Design of Auxiliary Decision-Making System for Power Grid Fault Disposal
}

\author{
Hao Chen ${ }^{1}$,Xinfan Jiang ${ }^{1}$,Dijun $\mathrm{Hu}^{1}$, Tao Wang ${ }^{1}$ and Fan Zhou ${ }^{1}$ \\ ${ }^{1}$ State Grid Hunan Electric Power Company, China
}

\begin{abstract}
Accident treatment is the most important part in the daily monitoring, and the improvement of the intelligent level is of great significance to improve the efficiency of scheduling accident and shorten the time of the accident. This paper puts forward a set of intelligent power grid accident treatment decision system, introduces the background of the system, the composition module and the realization of functions, to facilitate the operation of the operator to automatically identify the grid failure, isolation and recovery, reduce the pressure of staff, guarantee the safe and stable operation of the power grid.
\end{abstract}

\section{Introduction}

The safety, stability and economy of electric power system are the main objectives of power grid operation. In the modern power system, computer system provides the function of alarm and fault information collection, this undoubtedly improve the systematic level of the power grid operation, and provides the technical conditions for fault information. But on the other hand, under the state of emergency and fault, due to the lack of a unified comprehensive failure data processing and analysis tools, the influx of information has made it difficult for operators to accurately filter information and identify faults, the dispatcher was unable to accurately judge the failure, leading to miss the best time to dealing with accidents and cause an accident.

Document 1 and Document 2 put forward the key technical points of implementing the intelligent decision support system for incident handling based on the dispatching automation system. Among them, Document 1 proposes such typical applications as fault isolation and recovery, intelligent solution generation and coprocessing, thereby greatly improving the system's task handling capability and ensuring the safe and stable operation of the power grid.Typical applications as Document 2 refered,such as techniques of smart antierror strategy, information flow control, clearly the basic architecture of the system and the functional requirements, and studied the accident disposal program generation, intelligent load shedding brownouts, power transfer intelligent decision-making system and so on, which can provide dispatchers with preconceived accident anticipation and simulated training platform for anti-accident drills during the normal operation of the power grid. Document 3 adapt a common fault location method based on the EMS platform for incident handling intelligent decision support system, According to the grid operation mode, power flow control, transmission line capacity constraints and accident management regulations, the document proposes a recovery control strategy suitable for the Guangzhou power grid, which makes the system provide ancillary decision-making solutions in real-time and research states, and making it convenient for dispatching operation personnel to carry out accident anticipation disposal and real-time recovery control, reduce personnel pressure, ensure safe and stable operation of power grid. In addition, document 5 presents a holistic framework for online incident-handling decision support for large power grids. The study also includes five aspects of comprehensive information support platform, prior risk perception, online fault diagnosis, post-event decision support and visual display.

The basis of the decision support system for power grid accident is the construction of an integrated information platform. The key is to establish a tracking and processing mechanism for all aspects of risk monitoring and analysis beforehand, during and after the event. Finally, visualization techniques are needed to create a panoramic visualization of incident handling and to improve accident handling emergency decisionmaking ability. In the past, the researches on the auxiliary decision-making of accident handling basically focused on one aspect, lacking the holistic solution. The typical representatives include the White Paper on "21st Century EMS Architecture" proposed by VLGO, focusing on the construction of basic information platform. The advanced control center (AC2) being developed by PJM, a regional power market operator in the United States, focuses on the electricity market and the platform architecture. 
Therefore, there is an urgent need for an effective intelligent decision-making system for accident handling, control the operation of the power grid in real time by automated technology, timely detect, rapid diagnose and eliminate the problems in time, improve its automation level of scheduling and dealing with the accident, ensure the security of power system operation.

\section{The basic framework of auxiliary decision-making system of power grid accident management}

The intelligent auxiliary decision-making system of power grid accident management is located in the EMS platform, which shows the characteristics of the accident treatment of the regional power grid and provides intelligent technical support for fault diagnosis and method of power grid operation condition. Once in the event of an accident abnormality, the simple and complex faults in the power grid can be analyzed by capturing the real-time switching variable tidal current data and protecting the security information of the self-assembly device, so that the fault diagnosis and disposal scheme can be realized quickly and accurately, reduce the risk of misplaced and misuse, improve the efficiency and accuracy of accident handling. The system functional architecture of the auxiliary decision system is shown in Fig 1.

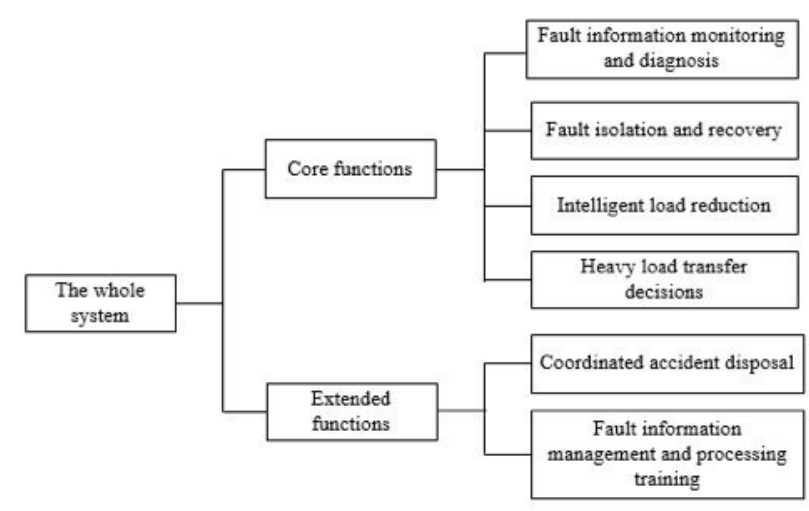

Figure 1. Schematic diagram of the functional architecture ofthe auxiliary decision-making system for power grid accident processing

The system requires the configuration of special accident handling assistant decision-making main server, through the configuration data interface, server and SCADA system can receive and monitor the power grid operation information in time. During the operation of the system, initiate different work which is responsible for the data flow control, fault diagnosis and fault disposal, from the application client we can view all kinds of information related to the fault.

Software design is based on the scheduling automation EMS platform, using independent application design and database design, we can fully share the SCADA various data, including real-time data and background parameters. The system can adapt to the
SCADA system of different manufacturers to ensure the reliability of the data source. When the user switches the SCADA module of different manufacturers, the auxiliary decision system can still obtain the basic information reliably. The data flow of SCADA is carried out in a security configuration, so that the data can be read from SCADA module in one way, which will not affect the stability and security of the program and data of SCADA module.

\section{The core function of auxiliary decision- making system for power grid accident handling}

\subsection{Fault information monitoring and diagnosis}

\subsubsection{Fault information detection}

After obtaining three remote signals, protection signal and other information from the EMS system SCADA, security and other modules, use data mining technology for data filtering, removing error duplication information, and normal operation information not related to the accident, adding missing fault information, to ensure that troubleshooting has a comprehensive data base. The detection of auxiliary decision system is reflected in two aspects. In the normal operation of the grid, the auxiliary decision system continues to carry out data detection, analyze and accumulate the various real-time information of SCADA module, identify normal operation information, debugging information and redundant information. When capture to the information which conforms to the characteristics of fault, including accident alarm message, switch shift, relay protection action information, immediately use data mining module for signal filtering and completion, after pretreatment, sent to the fault analysis module.

\subsubsection{Abnormal alarm of power grid}

The auxiliary decision system can monitor the operation condition of the power grid in real time. Once the discovery of cross-section, line, the main transformer close to the operating limit or the more limited, by issuing an alarm message, automatically pop-up site map or the whole network trend map, abnormal equipment flashing means to remind the dispatcher processing restrictions on developments, ensure that the duty dispatcher does not ignore the monitoring of the running device because he is too focused on a particular detail during the event.

\subsubsection{Comprehensive analysis of power grid faults}

After the grid failure actually happens, the fault analysis module automatically triggers. The fault analysis module uses the switch to change the information and protection action information, based on hierarchical intelligent technology to quickly locate the power outage range and 
lock the fault device, in a few seconds to complete the accident express, show to the dispatcher including fault equipment (when can not determine the only faulty equipment just provide the most likely suspicious equipment), power outage range, involving the power supply users, important users, reloading equipment list, and gives a preliminary evaluation of the switch to protect the action of the self-device.

Auxiliary decision system has two modes: real-time state and research state. Real-time state is based on SCADA information to trigger, all-weather automatic operation. The research state is mainly used when the basic data missing is too serious, dispatchers directly use the system to generate a fault handling program after setting up faulty device, or when the system is troublefree, the dispatcher anticipate the accident.

\subsection{Fault isolation and recovery}

In the operation of power grid, the fault is more obvious, but there will be a small number of permanent failure, in order to prevent the permanent damage to the power equipment, classify the type of fault and make the appropriate fault isolation and recovery operations will be necessary.

The operation process of fault isolation firstly is operational environment preparation, enable the relevant data monitoring procedures, monitor data ready in place. The second is real-time access to fault data, the data acquisition process should be detailed scan each smaller sub-module will produce the corresponding fault information, and according to the corresponding data analysis algorithm to determine whether the failure to achieve the conditions of the higher, only when more than a certain threshold, immediately start the automatic reporting mechanism for data reporting. Finally, through the intelligent network topology analysis, automatically generate the corresponding accident handling and disposal program, and use pre-set isolation program to complete the fault isolation operation and complete the failure of the disposal process, so as to effectively complete the fault of the rapid isolation and rapid recovery of pressure load and other operations.

\subsection{Intelligent load reduction}

Intelligent load shedding power module is mainly to complete the main transformer, cross-section is more than expected threshold, when the phenomenon of low frequency, low voltage occur, the original automatic device will automatically eliminate in a short period of time, and thus lose the safety of power grid security possibilities, can not make stable and safe operation of the grid. If the grid failure is to N-2 it will likely lead to serious failures occur, in order to prevent the occurrence of such events, generally use the operation strategy of automatic processing, when the corresponding malfunction occurs, the third grid security line will be activated immediately to protect and run itself, thus increasing the security and stability of the power grid.

\subsection{Heavy load transfer decisions}

In order to improve the reliability of urban power grid, the research object of emergency control of power grid accident management will not only be $10 \mathrm{KV}$ distribution lines, but also extended to $220 \mathrm{kV}, 110 \mathrm{kV}$ system and substation $10 \mathrm{kV}$ bus, and direct access to the above voltage level of distribution power supply. The urban power grid system which considers the main network, distribution network and distribution power supply can ensure the continuous power supply of important users in the emergency of the power grid or in the event of natural disasters.

\subsubsection{0kV line overload transfer}

$110 \mathrm{kV}$ line overload first according to the size of the overload to determine whether the load on the $110 \mathrm{kV}$ line may normal transfer, if can not transfer, then give the corresponding tips, if can transfer, give the transfer program, such as closing the corresponding mother joint switch and other operational programs, while the trend to check to determine whether the program will cause the equipment overload. $110 \mathrm{kV}$ line overload transfer based on its current operation mode may be normal operation mode, or may be a series operation mode.

For the normal operation mode, as shown in Figure 2 below, if L3 overload, through closing 7DL, disconnecting the $6 \mathrm{DL}$ to check the load transfer and the corresponding overload check.

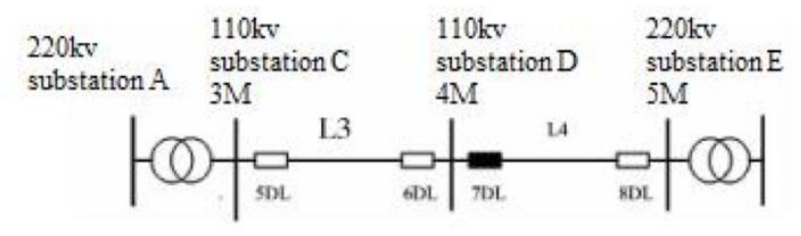

Figure 2. $110 \mathrm{kV}$ line common operation mode

For the series power supply mode, as shown in Figure 3 , if the L1 overload, the program may be given, including closing 7DL and disconnecting 6DL, or closing 7DL and disconnecting 4DL and other operational programs.

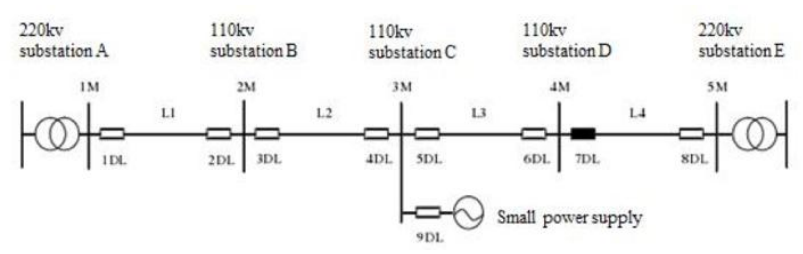

Figure 3. $110 \mathrm{kV}$ line multi-level serial supply operation mode

\subsubsection{0kV line overload transfer}

$220 \mathrm{kV}$ line is usually running by ring network connection, the given principle of overload transfer program is based on the power flow to first determine the $220 \mathrm{kV}$ plant of the line, the searched $110 \mathrm{kV}$ line outlet load of $220 \mathrm{kV}$ carry out the transfer according to $220 \mathrm{kV}$ main transformer overload transfer mode, while the trend check. 


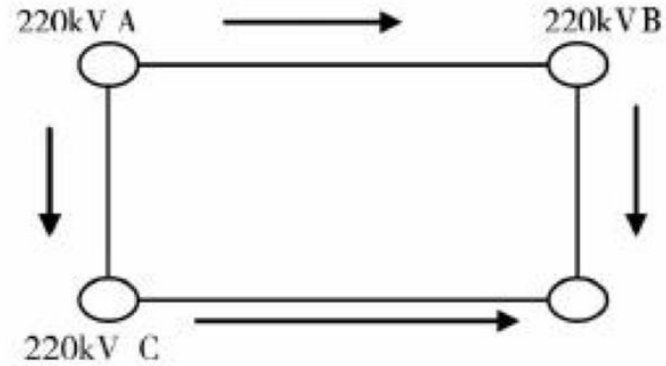

Figure 4. $220 \mathrm{kV}$ line ring network operation mode

If the line between $220 \mathrm{kV}$ A station and $220 \mathrm{kV} \mathrm{C}$ station is overloaded, as shown in fig.4, the corresponding $110 \mathrm{kV}$ line transfer in $220 \mathrm{kVC}$ station will be preferred. If there is no plan, then the corresponding $110 \mathrm{kV}$ line load will be considered in the transfer of $220 \mathrm{kVD}$ station. The transfer load is calculated according to the injection sensitivity of node B and D station, and the load transfer is calculated accordingly.

\subsubsection{0kV main transformer overload transfer}

The line of the middle - voltage side of $220 \mathrm{kV}$ is combined to obtain the optimal combination line group to complete the transfer and to conduct the tidal current check. The main switching strategy is: $110 \mathrm{kV}$ contact line direct revolution, multi-level chain structure $110 \mathrm{kV}$ circuit switching, substation $110 \mathrm{kV}$ bus transmission, etc.

\section{The expansion functions of power grid accident processing intelligent auxiliary decision system}

\subsection{Coordinated accident disposal}

After the accident, decision-making system should be able to intelligently generate the list of key steps of accident treatment, and can intelligently divide work according to the number and post of duty dispatcher, each task can be completed in the step table, Other dispatchers can get a clear picture of the progress of the work done by the current dispatchers by looking at the real-time refresh of the task table, and can then be divided according to the schedule.

\subsection{Fault information management and processing training}

The auxiliary decision system provides a full range of fault case information management functions. Include event saving, retrieval, case library update, process report presentation and printout. You can retrieve the accident express, troubleshooting programs, processing information and so on.

The auxiliary decision system is an important tool for the training of dispatcher accident handling in the absence of an accident abnormality. Through the data interaction with the DTS module, the dispatcher in the auxiliary decision-making system to manually design the accident handling program is the process of DTS lesson plan training. On the other hand, demonstrating typical accident disposal cases in the system and giving a hint at the key processing node, can be more intuitive to the dispatcher who needs training, and can significantly improve the learning effect than simply reading the text system.

\section{Conclusion}

Based on the characteristic information of power grid production information, this paper designs the basic structure and basic functional requirements of the auxiliary decision system of power grid accident disposal. The system greatly improves the dispatcher's ability to deal with the power grid accident, reduces the decisionmaking time of the accident processing, makes the analysis of the grid accident gradually from the empirical type to the analysis, intelligent, improve the grid independent support and self-fast resilience after the accident. On the other hand, after the system is put into operation, the dispatcher enhances the understanding of the current operation mode of the power grid by simulating the operation of the power supply load and the overload load transfer, so that the power grid can operate more efficiently and reliably.

\section{References}

1. Liu Yang. Design and implementation of power grid incident management system[D]. University of Electronic Science and Technology of China, 2014.M. Ben Rabha, M.F. Boujmil, M. Saadoun, B. Bessaïs, Eur. Phys. J. Appl. Phys. (to be published)

2. Zhang Weihua. Research on intelligent auxiliary decision-making system of power grid accident handling [D]. South China University of Technology, 2011.

3. Yang Zeng, Liu Yuquan, Tan Xilin, Pan Kaiyan. An intelligent auxiliary system based on EMS platform

4. Yunnan Electric Power Technology, 2013,41 (05): 51-53.

5. Zhang Tao, Zhang Bin, Li Zhiqiang, Huang Xiangdong. Application of intelligent grid accident auxiliary decision system in scheduling [J]. Power Supply, 2009,26(05):26-28+36.

6. Shan Xin. Design and implementation of the auxiliary decision-making system for online accident management of power grid [A]. China electrical engineering institute. Proceedings of the annual meeting of the China institute of electrical engineering in 2013 [C]. China electrical engineering society: 2013:6.

7. Yanru Sun,Wei He. Online security risk prevention and control of power grid operation based on Smart Grid Dispatching Technology[J/OL]. Modern Industrial Economy and Informationization, 2017 (21) :55-56+61

8. Nanrong He. Design and implementation of power grid accident warning and disposal management system[D].Jilin University,2016. 
9. Xiangyu Zhao, Chengliang Wang, Gang Yao,Xiang Fan,Guojiang Xiong,Xu Zhang,Luofang Zhu ,Xiaoxu Huang.Design and application of online accident risk early warning system for power grid based on South network accident regulation[J]. Electric Power,2016,49(S1):62-66.
10. Chengbin Gao. Research and system development of power grid operation risk assessment and accident warning[D].North China Electric Power University,2015. 